\title{
A COMPARISON RESULT FOR THE OSCILLATION OF DELAY DIFFERENTIAL EQUATIONS
}

\author{
G. LADAS, C. QIAN, AND J. YAN
}

(Communicated by Kenneth R. Meyer)

ABSTRACT. We obtain a comparison result for the oscillation of all solutions of the equation

$$
\dot{y}(t)+\sum_{i=1}^{n} q_{i}(t) y\left(t-\sigma_{i}(t)\right)=0
$$

in terms of the oscillation of all solutions of the equation

$$
\dot{x}(t)+\sum_{i=1}^{n} p_{i}(t) x\left(t-\tau_{i}(t)\right)=0
$$

under appropriate hypotheses on the asymptotic behavior of the quotients

$$
p_{i}(t) / q_{i}(t) \text { and } \tau_{i}(t) / \sigma_{i}(t) \text { for } i=1,2, \ldots, n .
$$

An application to the oscillation of the nonautonomous delay-logistic equation is given.

\section{INTRODUCTION}

Let $\mathbf{R}$ denote the set of real numbers and let $\mathbf{R}^{+}$denote the set of nonnegative real numbers. Consider the delay differential equations

$$
\dot{x}(t)+\sum_{i=1}^{n} p_{i}(t) x\left(t-\tau_{i}(t)\right)=0
$$

and

$$
\dot{y}(t)+\sum_{i=1}^{n} q_{i}(t) y\left(t-\sigma_{i}(t)\right)=0,
$$

where for each $i=1,2, \ldots, n$,

$$
p_{i}, \tau_{i} \in C\left[\left[t_{0}, \infty\right), \mathbf{R}^{+}\right], \quad \lim _{t \rightarrow \infty}\left[t-\tau_{i}(t)\right]=\infty
$$

Received by the editors December 6, 1989.

1980 Mathematics Subject Classification (1985 Revision). Primary 34K15.

The second author worked on this paper while on leave from the Department of Mathematics, Yangzhou Teachers College, Yangzhou, Jiangsu, People's Republic of China.

The third author worked on this paper while on leave from the Department of Mathematics, Shanxi University, Taiyuan, Shanxi, People's Republic of China. 
and

$$
q_{i}, \sigma_{i} \in C\left[\left[t_{0}, \infty\right), \mathbf{R}^{+}\right], \quad \lim _{t \rightarrow \infty}\left[t-\sigma_{i}(t)\right]=\infty .
$$

We obtain a comparison result for the oscillation of all solutions of (2) in terms of the oscillation of all solutions of (1) provided that the following hypothesis is satisfied:

For each $i=1,2, \ldots, n$ the zeros $z_{i}$ of $p_{i}$ and $q_{i}$ and the zeros $\tilde{z}_{i}$ of $\tau_{i}$ and $\sigma_{i}$ are the same with the same multiplicities and the following limits exist:

$$
\alpha_{i}=\lim _{\substack{t \rightarrow \infty \\ t \in\left[t_{0}, \infty\right)-z_{i}}} \frac{p_{i}(t)}{q_{i}(t)} \text { and } \beta_{i}=\lim _{\substack{t \rightarrow \infty \\ t \in\left[t_{0}, \infty\right)-\tilde{z}_{i}}} \frac{\tau_{i}(t)}{\sigma_{i(t)}} .
$$

The main result that we establish in $\S 3$ is the following:

Theorem 1. Assume that (3), (4), and (5) hold. Then the following statements are true:

(a) Assume that

$$
\alpha_{i} \leq 1 \text { and } \beta_{i} \leq 1 \text { for } i=1,2, \ldots, n
$$

and that every solution of (1) oscillates. Then every solution of (2) also oscillates.

(b) Assume that

$$
\alpha_{i}=\beta_{i}=1 \text { for } i=1,2, \ldots, n .
$$

Then every solution of (1) oscillates if and only if every solution of (2) oscillates.

In $\S 4$ we present an application of the comparison theorem to the oscillation of all positive solutions of the nonautonomous delay-logistic equation

$$
\dot{N}(t)=r(t) N(t)\left[1-\frac{N(t-\tau(t))}{K}\right]
$$

about the positive equilibrium $K$.

For any $T \geq t_{0}$ we define

$$
T_{-1}=\min _{1 \leq i \leq n}\left\{\inf _{t \geq T}\left\{t-\tau_{i}(t)\right\}\right\} .
$$

By a solution of (1) we mean a function $x$ that is continuously differentiable on $[T, \infty)$ for some $T \geq t_{0}$, which is defined and continuous on $\left[T_{-1}, \infty\right)$ and which satisfies (1) for $t \geq T$.

With (1) and a given "initial point" $T \geq t_{0}$, one associates an "initial condition" of the form

$$
x(t)=\phi(t) \text { for } T_{-1} \leq t \leq T,
$$

where $\phi \in C\left[\left[T_{-1}, T\right], \mathbf{R}\right]$ is a given "initial function." Then the IVP (1) and (8) has a unique solution $x$ valid on $[T, \infty)$. That is, $x$ is defined and continuous on $\left[T_{-1}, \infty\right), x$ is continuously differentiable on $[T, \infty), x$ satisfies (8), and $x$ satisfies (1) for $t \geq T$ (see Hale [2]).

As usual, a solution of (1) is called oscillatory if it has arbitrarily large zeros. Otherwise the solution is called nonoscillatory. Finally, a function $N(t)$ is said to oscillate about $K$ if $N(t)-K$ has arbitrarily large zeros. 
In the sequel, unless otherwise specified, when we write a functional inequality we will assume that it holds for all sufficiently large $t$.

\section{SOME BASIC LEMMAS}

In this section we present two lemmas that are interesting in their own rights and will be useful in the proof of Theorem 1 in $\S 3$.

With (1) and for every point $T \geq t_{0}$ we associate the following sequence of functions:

$$
u_{0}(t)=0 \text { for } t \geq T_{-1}
$$

and for $k=1,2, \ldots$,

$$
u_{k}(t)=\left\{\begin{array}{l}
0 \quad \text { for } T_{-1} \leq t<T, \\
\sum_{i=1}^{n} p_{i}(t) \exp \left(\int_{t-\tau_{i}(t)}^{t} u_{k-1}(s) d s\right) \quad \text { for } t \geq T,
\end{array}\right.
$$

where $T_{-1}$ is as defined by (7).

Lemma 1. Assume that (3) holds. Then the following statements are equivalent.

(a) Equation (1) has a nonoscillatory solution.

(b) There exists $T \geq t_{0}$ such that the sequence $\left\{u_{k}(t)\right\}$ which is defined by (9) converges pointwise to a finite limit for each $t \geq T$.

Proof. (a) $\Rightarrow$ (b). Clearly, we can assume that (1) has a solution $x(t)$ such that $x(t)>0$ for $t \geq t_{1} \geq t_{0}$. Choose $T \geq t_{1}$ such that $T_{-1} \geq t_{1}$. Set

$$
u(t)=-\frac{\dot{x}(t)}{x(t)} \quad \text { for } t \geq T_{-1} \text {. }
$$

Then for $t \geq T, u(t) \geq 0$. By integrating (10) we find

$$
x(t)=x\left(t-\tau_{i}(t)\right) \exp \left(\int_{t-\tau_{i}(t)}^{t} u(s) d s\right) .
$$

From (1), (10), and (11) it follows that

$$
u(t)=\sum_{i=1}^{n} p_{i}(t) \exp \left(\int_{t-\tau_{i}(t)}^{t} u(s) d s\right) \text { for } t \geq T
$$

An induction argument using (9) and (12) yields that

$$
u_{k}(t) \leq u_{k+1}(t) \leq u(t) \text { for } t \geq T \text { and } k=0,1,2, \ldots
$$

from which it follows that the sequence $\left\{u_{k}(t)\right\}$ converges pointwise to a finite limit for each $t \geq T$.

(b) $\Rightarrow($ a) . Clearly

$$
u_{k}(t) \leq u_{k+1}(t) \text { for } t \geq T_{-1} \text { and } k=0,1,2, \ldots
$$

Let

$$
u(t)=\lim _{t \rightarrow \infty} u_{k}(t) \quad \text { for } t \geq T_{-1} \text {. }
$$

Then, by Lebesgue's monotone convergence theorem, $u(t)$ satisfies (12). Finally, observe by direct substitution that

$$
x(t)=\exp \left(-\int_{T_{-1}}^{t} u(s) d s\right), \quad t \geq T_{-1}
$$


is a positive solution of (1). The proof is complete.

The next lemma deals with delay differential equations that involve a parameter $\mu$ in the delays and the coefficients. It states that, under appropriate hypotheses, the set of all points $\mu$ for which every solution of the equation

$$
\dot{y}(t)+\sum_{i=1}^{n} p_{i}(t, \mu) y\left(t-\tau_{i}(t, \mu)\right)=0
$$

oscillates is open.

Lemma 2. Let $M$ be an open subset of $\mathbf{R}$. Assume that for each $i=1,2, \ldots, n$ and for each fixed $\mu \in M$, the functions $p_{i}(\cdot, \mu)$ and $\tau_{i}(\cdot, \mu)$ are nonnegative and continuous on $\left[t_{0}, \infty\right)$ and

$$
\lim _{t \rightarrow \infty}\left[t-\tau_{i}(t, \mu)\right]=\infty
$$

Also assume that for each $i=1,2, \ldots, n$ and for fixed $t \geq t_{0}$ the functions $p_{i}(t, \cdot)$ and $\tau_{i}(t, \cdot)$ are continuous on $M$. Let $S$ be the set of all $\mu \in M$ such that every solution of $\left(13_{\mu}\right)$ oscillates. Then $S$ is an open subset of $M$.

Proof. For any $\mu \in M$ and for any $T \geq t_{0}$ we define the following sequence of functions:

$$
u_{0}(t, \mu)=0 \text { for } t \geq T_{-1}(\mu)
$$

and for $k=1,2, \ldots$

$$
u_{k}(t, \mu)=\left\{\begin{array}{l}
0 \quad \text { for } T_{-1}(\mu) \leq t<T, \\
\sum_{i=1}^{n} p_{i}(t, \mu) \exp \left(\int_{t-\tau_{i}(t, \mu)}^{t} u_{k-1}(s, \mu) d s\right) \quad \text { for } t \geq T,
\end{array}\right.
$$

where

$$
T_{-1}(\mu)=\min _{1 \leq i \leq n}\left\{\inf _{t \geq T}\left\{t-\tau_{i}(t, \mu)\right\}\right\} \text {. }
$$

By induction, it is easy to see that $u_{k}(t, \mu)$ for $k=1,2, \ldots$ are continuous on $[T, \infty)$ with respect to $t$ and on $M$ with respect to $\mu$.

Suppose that $\left(13_{\mu_{0}}\right)$ (that is $\left(13_{\mu}\right)$ with $\mu$ replaced by $\left.\mu_{0}\right)$ is oscillatory for some $\mu_{0} \in M$. Then by Lemma 1 for any sufficiently large $T_{1} \geq T$ there exists a $t^{*} \geq T_{1}$ such that

$$
\lim _{k \rightarrow \infty} u_{k}\left(t^{*}, \mu_{0}\right)=\infty
$$

Hence, for any large constant $m>0$ there exists $K$ such that for any $k>K$

$$
u_{k}\left(t^{*}, \mu_{0}\right)>m \text {. }
$$

Since $u_{k}\left(t^{*}, \mu\right)$ is continuous with respect to $\mu$, for some $k_{0}>K$ there is a neighborhood $\delta\left(\mu_{0}\right) \subset M$ of $\mu_{0}$ such that

$$
u_{k_{0}}\left(t^{*}, \mu\right)>m \text { for any } \mu \in \delta\left(\mu_{0}\right) \text {. }
$$

On the other hand from the monotonicity of $u_{k}\left(t^{*}, \mu\right)$ with respect to $k$, we see that

$$
u_{k}\left(t^{*}, \mu\right)>m \text { for any } k \geq k_{0} \text { and } \mu \in \delta\left(\mu_{0}\right) \text {. }
$$

Clearly, this implies that

$$
\lim _{t \rightarrow \infty} u_{k}\left(t^{*}, \mu\right)=\infty \text { for } \mu \in \delta\left(\mu_{0}\right) .
$$

Therefore by Lemma $1,\left(13_{\mu}\right)$ is oscillatory for any $\mu \in \delta\left(\mu_{0}\right)$. This implies that $S$ is an open set and the proof is complete.

The following result is an immediate consequence of Lemma 2. 
Corollary 1. Let $\left\{\varepsilon_{k}\right\}$ be a sequence of positive numbers such that $\lim _{k \rightarrow \infty} \varepsilon_{k}=0$ and such that for every $i=1,2, \ldots, n$ and every $k \geq 1, p_{i}-\varepsilon_{k} \geq 0, \tau_{i}-\varepsilon_{k} \geq 0$ and the delay differential equation:

$$
\dot{y}(t)+\sum_{i=1}^{n}\left(p_{i}-\varepsilon_{k}\right) y\left(t-\left(\tau_{i}-\varepsilon_{k}\right)\right)=0, \quad t \geq t_{0}
$$

has a nonoscillatory solution. Then the delay differential equation:

$$
\dot{y}(t)+\sum_{i=1}^{n} p_{i} y\left(t-\tau_{i}\right)=0
$$

also has a nonoscillatory solution.

\section{PROOF OF THE COMPARISON THEOREM}

Before we present the proof of Theorem 1 we need the following corollary of Lemma 1. This result is, in itself, an important comparison theorem for oscillations and was first established (in a slightly weaker form) by different techniques, by Kwong and Patula [5].

Corollary 2. Assume that (3) and (4) hold and that for each $i=1,2, \ldots, n$,

$$
p_{i}(t) \leq q_{i}(t) \text { and } \tau_{i}(t) \leq \sigma_{i}(t) \quad \text { for } t \geq t_{0} .
$$

Suppose that every solution of (1) oscillates. Then every solution of (2) also oscillates.

Proof. For every $T \geq t_{0}$, set

$$
v_{0}(t)=0 \text { for } t \geq T_{-1}^{*},
$$

and for $k=1,2, \ldots$, define

$$
v_{k}(t)=\left\{\begin{array}{l}
0 \text { for } T_{-1}^{*} \leq t<T, \\
\sum_{i=1}^{n} q_{i}(t) \exp \left(\int_{t-\sigma_{i}(t)}^{t} v_{k-1}(s) d s\right) \quad \text { for } t \geq T,
\end{array}\right.
$$

where

$$
T_{-1}^{*}=\min _{1 \leq i \leq n}\left\{\inf _{t \geq T}\left\{t-\sigma_{i}(t)\right\}\right\} .
$$

Assume, for the sake of contradiction, that (2) has a nonoscillatory solution. Then by Lemma 1 there exists $T \geq t_{0}$ such that the sequence $\left\{v_{k}(t)\right\}$ converges pointwise to a finite limit for $t \geq T$. Clearly, the sequence $\left\{u_{k}(t)\right\}$ which is defined by (9) also converges pointwise to a finite limit for each $t \geq T$ since $u_{k}(t) \leq v_{k}(t)$. Then by Lemma 1 again, we see that (1) has a nonoscillatory solution. This is a contradiction and the proof is complete.

Corollary 3. Suppose that (3) holds and that

(14) $\liminf _{t \rightarrow \infty} p_{i}(t) \geq \tilde{p}_{i}>0$ and $\liminf _{t \rightarrow \infty} \tau_{i}(t) \geq \tilde{\tau}_{i}>0$ for $i=1,2, \ldots, n$.

If every solution of the differential equation

$$
\dot{y}(t)+\sum_{i=1}^{n} \tilde{p}_{i} y\left(t-\tilde{\tau}_{i}\right)=0
$$


is oscillatory, then every solution of (1) is also oscillatory.

Proof. By Lemma 2 we see that there exists a constant $\tilde{\varepsilon}>0$ such that

$$
\tilde{p}_{i}-\tilde{\varepsilon}>0 \text { and } \tilde{\tau}_{i}-\tilde{\varepsilon}>0 \text { for } i=1,2, \ldots, n
$$

and such that every solution of the differential equation:

$$
\dot{y}(t)+\sum_{i=1}^{n}\left(\tilde{p}_{i}-\tilde{\varepsilon}\right) y\left(t-\left(\tilde{\tau}_{i}-\tilde{\varepsilon}\right)\right)=0
$$

is oscillatory. By (14) we see that eventually

$$
p_{i}(t) \geq \tilde{p}_{i}-\tilde{\varepsilon} \text { and } \tau_{i}(t) \geq \tilde{\tau}_{i}-\tilde{\varepsilon} \quad \text { for } i=1,2, \ldots, n .
$$

Hence by Corollary 2, every solution of (1) is also oscillatory. The proof is complete.

Proof of Theorem 1. (a) For every $\mu \in(0,1)$ and for each $i=1,2, \ldots, n$ we eventually have

$$
(1-\mu) p_{i}(t) \leq q_{i}(t) \text { and }(1-\mu) \tau_{i}(t) \leq \sigma_{i}(t) .
$$

It also follows from Lemma 2 and the fact that every solution of $(1)$ is oscillatory, that for $\mu \in(0,1)$ and sufficiently small, every solution of the equation

$$
\dot{z}(t)+\sum_{i=1}^{n}(1-\mu) p_{i}(t) z\left(t-(1-\mu) \tau_{i}(t)\right)=0
$$

is also oscillatory. In view of (15), Corollary 2 now implies that every solution of (2) is oscillatory.

(b) The proof of this statement is a consequence of (a) and will be omitted.

The next corollary is an immediate consequence of Theorem 1. It also extends and improves in a certain way Theorems 2 and 5 of [4] which for the sake of comparison we summarize below.

Theorem A. Assume that for $j=1,2, \ldots, n$,

$$
\tau_{j} \in[0, \infty) \text { and } p_{j} \in C\left[\left[t_{0}, \infty\right), \mathbf{R}^{+}\right] .
$$

(a) If

$$
\lim _{t \rightarrow \infty} p_{j}(t)=\tilde{p}_{j}
$$

and every solution of the delay equation:

$$
\dot{x}(t)+\sum_{j=1}^{n} \tilde{p}_{j} x\left(t-\tau_{j}\right)=0
$$

is oscillatory then every solution of the equation:

$$
\dot{y}(t)+\sum_{j=1}^{n} p_{j}(t) y\left(t-\tau_{j}\right)=0
$$

is also oscillatory.

(b) If

$$
p_{j}(t) \leq \tilde{p}_{j}
$$


and (17) has a nonoscillatory solution, then (18) also has a nonoscillatory solution.

(c) If (16) and (19) are satisfied then every solution of (17) is oscillatory if and only if every solution of (18) is oscillatory.

Corollary 4. Assume that (3) holds and that there exist positive numbers $\tilde{p}_{i}$ and $\tilde{\tau}_{i}$ such that

$$
\lim _{t \rightarrow \infty} p_{i}(t)=\tilde{p}_{i} \quad \text { and } \quad \lim _{t \rightarrow \infty} \tau_{i}(t)=\tilde{\tau}_{i} \quad \text { for } i=1,2, \ldots, n .
$$

Then (1) is oscillatory if and only if the "limiting" equation

$$
\dot{y}(t)+\sum_{i=1}^{n} \tilde{p}_{i} y\left(t-\tilde{\tau}_{i}\right)=0
$$

is oscillatory, that is (see [1]), if and only if its characteristic equation

$$
\lambda=\sum_{i=1}^{n} \tilde{p}_{i} e^{\lambda \tilde{\tau}_{i}}
$$

has no real roots.

Corollary 5. Assume that $p, \tau \in C\left[\left[t_{0}, \infty\right), \mathbf{R}^{+}\right]$and that there exist positive constants $\tilde{p}$ and $\tilde{\tau}$ such that

$$
\lim _{t \rightarrow \infty} p(t)=\tilde{p} \quad \text { and } \quad \lim _{t \rightarrow \infty} \tau(t)=\tilde{\tau} .
$$

Then every solution of the delay differential equation:

$$
\dot{y}(t)+p(t) y(t-\tau(t))=0
$$

is oscillatory if and only if

$$
\lim _{t \rightarrow \infty} \int_{t-\tau(t)}^{t} p(s) d s>\frac{1}{e} .
$$

Proof. By Corollary 4, (21) is oscillatory if and only if

$$
\lambda=\tilde{p} e^{\lambda \tilde{\tau}}
$$

has no real roots. One can also see that (23) has no real roots if and only if

$$
\tilde{p} \tilde{\tau}>1 / e \text {. }
$$

Clearly if (20) holds, (24) is equivalent to (22).

Remark 1. It is well known (see [3]) that a sufficient condition for (21) to have a nonoscillatory solution is that eventually,

$$
\int_{t-\tau(t)}^{t} p(s) d s \leq \frac{1}{e} .
$$

One can see from Corollary 5 that the condition (25) is not necessary for (21) to have a nonoscillatory solution.

\section{OSCILlations IN A NONAUTONOMOUS DELAY-LOGISTIC EQUATION}

In this section we will use Theorem 1 to obtain sufficient conditions for the oscillation of all positive solutions of the nonautonomous delay-logistic equation

$$
\dot{N}(t)=r(t) N(t)\left[1-\frac{N(t-\tau(t))}{K}\right],
$$


where

$$
r, \tau \in C\left[\left[t_{0}, \infty\right), \mathbf{R}^{+}\right], \quad K \in(0, \infty), \quad \text { and } \quad \lim _{t \rightarrow \infty}(t-\tau(t))=\infty .
$$

Our result extends the following recent result of Zhang and Gopalsamy [6].

Theorem B. Assume that (27) holds,

$$
\int_{0}^{\infty} r(s) d s=\infty
$$

and that

$$
\liminf _{t \rightarrow \infty} \int_{t-\tau(t)}^{t} r(s) d s>\frac{1}{e} .
$$

Then every positive solution of (26) oscillates about the positive equilibrium $K$.

We will prove that the conclusion of Theorem B remains true, if condition (29) is replaced by the following weaker hypothesis:

(H) Every solution of the linear delay equation

$$
\dot{y}(t)+r(t) y(t-\tau(t))=0
$$

oscillates about zero.

With (26) one associates an initial condition of the form

$$
N(t)=\phi(t) \text { for } T_{-1} \leq t \leq 0,
$$

where

$$
\phi \in C\left[\left[T_{-1}, 0\right], \mathbf{R}^{+}\right] \text {and } \phi(0)>0
$$

with

$$
T_{-1}=\inf _{t \geq 0}[t-\tau(t)]
$$

Then by the method of steps, the initial value problem (26) and (31) has a unique solution $N(t)$ which is valid for $t \geq 0$. We will only consider those solutions $N(t)$ which are positive. Note that such solutions exist because if $\phi(0)>0$, then $N(t)>0$ for $t \geq 0$. The change of variables

$$
N(t)=K e^{x(t)}
$$

reduces (26) to the delay equation

$$
\dot{x}(t)+r(t)\left[e^{x(t-\tau(t))}-1\right]=0 .
$$

Clearly, $N(t)$ oscillates about $K$ if and only if $x(t)$ oscillates about zero. Now we are ready to establish the following extension of Theorem $B$.

Theorem 2. Assume that (27) and (28) hold and that every solution of (30) oscillates. Then every positive solution of (26) oscillates about the steady state $K$.

Proof. Otherwise (32) has a nonoscillatory solution $x(t)$. Then eventually,

$$
\begin{array}{llll}
\text { either } & x(t)>0 \text { and } & \dot{x}(t) \leq 0 \\
\text { or } & x(t)<0 \text { and } \dot{x}(t) \geq 0 .
\end{array}
$$


Thus the $\lim _{t \rightarrow \infty} x(t)$ exists and is a finite number $l$. From (32) and (28) it follows that $l=0$. Set

$$
R(t)=r(t) \frac{e^{x(t-\tau(t))}-1}{x(t-\tau(t))}
$$

and observe that $R(t)>0$, the functions $r$ and $R$ have the same set of zeros $z$,

$$
\lim _{\substack{t \rightarrow \infty \\ t \notin z}} \frac{r(t)}{R(t)}=1,
$$

and that $x(t)$ is a nonoscillatory solution of the equation

$$
\dot{z}(t)+R(t) z(t-\tau(t))=0 .
$$

It follows, by Theorem 1 and the hypothesis that every solution of $(30)$ oscillates, that every solution of (33) also oscillates. This contradicts the fact that $x(t)$ is a nonoscillatory solution of (33). The proof is complete.

\section{REFERENCES}

1. I. Györi and G. Ladas, Oscillation theory of delay differential equations with applications, Oxford Univ. Press (to appear).

2. J. K. Hale, Theory of functional differential equations, Springer-Verlag, New York, 1977.

3. R. G. Koplatadze and T. A. Chanturia, On oscillatory and monotonous solutions of first order differential equation with deviating argument, Differential'nye Uravnienija 18 (1982), 1464-1465. (Russian)

4. M. R. S. Kulenovic, G. Ladas, and A. Meimaridou, On oscillation of nonlinear delay differential equations, Quart. Appl. Math. 45 (1987), 155-164.

5. M. K. Kwong and W. T. Patula, Comparison theorems for first order linear delay equations, J. Differential Equations 70 (1987), 275-295.

6. B. G. Zhang and K. Gopalsamy, Oscillation and nonoscillation in a nonautonomous delaylogistic equation, Quart. Appl. Math. XLVI (1988), 267-273.

Department of Mathematics, The University of Rhode Island, Kingston, Rhode ISLAND 02881-0816 